\title{
NEW INVARIANT EINSTEIN METRICS ON GENERALIZED FLAG MANIFOLDS
}

\author{
ANDREAS ARVANITOYEORGOS
}

\begin{abstract}
A generalized flag manifold (or a Kählerian $C$-space) is a homogeneous space $G / K$ whose isotropy subgroup $K$ is the centralizer of a torus in $G$. These spaces admit a finite number of Kähler-Einstein metrics. We present new non-Kähler Einstein metrics for certain quotients of $U(n), S O(2 n)$ and $G_{2}$. We also examine the isometry question for these metrics.
\end{abstract}

\section{INTRODUCTION}

A metric $g$ on a Riemannian manifold $M$ is called an Einstein metric if $\operatorname{Ric}(g)=c g$ for some constant $c$. Einstein metrics are generally considered as privileged metrics on a given manifold [Be, pp. 1-5]. In this paper we present Einstein metrics for some examples of homogeneous spaces called generalized flag manifolds.

Let $G$ be a compact, connected and semisimple Lie group. A generalized flag manifold is a homogeneous space $M=G / K$ whose isotropy subgroup $K$ is the centralizer of a one-parameter subgroup $\exp t w$ of $G$. Here $w$ is an element in the Lie algebra $\mathfrak{g}$ of $G$. A generalized flag manifold is also referred to as a Kählerian $C$-space. We shall use this terminology from now on.

Equivalently, $M$ is the adjoint orbit $\operatorname{Ad}(G) w$ of $w$ under the action of the adjoint representation Ad of $G$ in $\mathfrak{g}$. It follows that the isotropy subgroup $K$ is the centralizer of a torus in $G$ [B-F-R, Wa]. The Kählerian $C$-spaces have been classified in [B-F-R and Wa]: there is an infinite sequence of homogeneous spaces for each of the classical Lie groups and a finite number for each of the exceptional ones.

It is known that each of these spaces admits a finite number of invariant complex structures [Alek-Pe, Be, B-F-R] and that there is a one-to-one correspondence between invariant complex structures (up to a sign) and invariant Kähler-Einstein metrics (up to a constant factor) on $M$ [Be, Alek]. It is also known [Wa-Zi] that for some of these spaces the normal metric is Einstein. In this paper we present new invariant Einstein metrics for certain examples of these Kählerian $C$-spaces. The presentation is organized as follows:

After reviewing in $\S 1$ the Lie algebraic description of the space $G / K$, we describe in $\S 2$ the so-called t-roots which will enable us to reduce the number of the unknowns in the Einstein equation. In $\S 3$ we describe $G$-invariant metrics

Received by the editors July 23, 1990 and, in revised form, March 21, 1991.

1980 Mathematics Subject Classification (1985 Revision). Primary 53C25, 53C30. 
on $M$ and in $\S 4$ we give a Lie algebraic expression for the Ricci tensor of such a metric, which is a corrected version of the one in [Alek]. In $\S 5$ we present the Einstein equation for the Kählerian $C$-spaces $S U(n) / S\left(U\left(n_{1}\right) \times \cdots \times U\left(n_{s}\right)\right)$, $S O(2 n) / U\left(n_{1}\right) \times \cdots \times U\left(n_{s}\right)$ and $G_{2} / U(2)$ and give solutions in some cases, and in $\S 6$ we examine which of the metrics obtained in $\S 5$ are isometric. We obtain

Theorem A. (1) $S U(n) / S\left(U\left(n_{1}\right) \times U\left(n_{2}\right) \times U\left(n_{3}\right)\right)$ admits precisely four invariant Einstein metrics (up to scale). Three of them are Kähler and one is non-Kähler.

(2) $S U(n) / S(U(1) \times \cdots \times U(1))$ (with $n$ factors equal to $U(1)$ ) admits precisely four invariant Einstein metrics if $n=3$ and at least $n ! / 2+1+n$ if $n \geq 4$. The $n ! / 2$ metrics are Kähler and the remaining $1+n$ are non-Kähler.

Theorem B. $S O(2 n) / U(m) \times U(m)(n=2 m)$ admits precisely ten invariant Einstein metrics. Six of them are non-Kähler.

Theorem C. $G_{2} / U(2)$ admits precisely three invariant Einstein metrics. One is Kähler and two are non-Kähler.

Explicit formulas for the above metrics can be found in $\S 5$ (Theorems 5, 7, 9, 11). Theorems $A(1)$ and $C$ have also been obtained by Masahiro Kimura [Ki], using the variational approach of Wang-Ziller [Wa-Zi].

The author wishes to express his sincere gratitude to Professor Martin Guest for his inspiring suggestions, as well as to the referee for his valuable comments.

\section{LIE THEORETIC DESCRIPTION OF $M=G / K$}

Since $G$ is semisimple and compact, the Killing form

$$
(X, Y)=\operatorname{tr} \operatorname{ad}(X) \operatorname{ad}(Y)
$$

of $\mathfrak{g}$ is nondegenerate and negative definite on $\mathfrak{g}$ thus giving rise to an orthogonal decomposition of $\mathfrak{g}$ as the direct sum $\mathfrak{g}=\mathfrak{k} \oplus \mathfrak{m}$. Here $\mathfrak{k}$ is the Lie algebra of $K$ and $\mathfrak{m}=\mathfrak{k}^{\perp}$.

Moreover the tangent space $T_{p} M$ can be identified with $\mathrm{m}$. This identification is given by

$$
X \mapsto X^{*}(p)=\left.\frac{d}{d t}(\exp t X \cdot p)\right|_{t=0}, \quad X \in \mathfrak{m}, p \in M
$$

For any vector space $V$ denote by $V^{\mathrm{C}}$ its complexification. We fix a Cartan subalgebra $\mathfrak{h}^{\mathbf{C}}$ of the complexified Lie algebra $\mathfrak{k}^{\mathbf{C}}$; then the Cartan decompositions of $\mathfrak{g}^{\mathbf{C}}$ and $\mathfrak{k}^{\mathbf{C}}$ are given as follows:

$$
\mathfrak{g}^{\mathbf{C}}=\mathfrak{h}^{\mathbf{C}}+\sum_{\alpha \in R} \mathfrak{g}^{(\alpha)}, \quad \mathfrak{k}^{\mathbf{C}}=\mathfrak{h}^{\mathbf{C}}+\sum_{\alpha \in R_{K}} \mathfrak{g}^{(\alpha)}, \quad \mathfrak{m}^{\mathbf{C}}=\sum_{\alpha \in R_{M}} \mathfrak{g}^{(\alpha)}
$$

where $R, R_{K}$ are the root systems of the pairs $\left(\mathfrak{g}^{\mathbf{C}}, \mathfrak{h}^{\mathbf{C}}\right)$ and $\left(\mathfrak{k}^{\mathbf{C}}, \mathfrak{h}^{\mathbf{C}}\right)$ respectively. The root system $R$ is decomposed as $R=R_{K} \cup R_{M}$ where $R_{M}$ is called the set of complementary roots. The spaces $\mathfrak{g}^{(\alpha)}$ are the 1-dimensional root spaces whose elements $X_{\alpha}$ are characterized by the equation $\left[H, X_{\alpha}\right]$ $=\alpha(H) X_{\alpha}, H \in \mathfrak{h}^{\mathbf{C}}$. We recall [Var, W] that for any root $\alpha$ we can choose elements $E_{\alpha} \in \mathfrak{g}^{(\alpha)}(\alpha \in R)$ which have the properties $\left(E_{\alpha}, E_{-\alpha}\right)=-1$, $\left[E_{\alpha}, E_{-\alpha}\right]=-H_{\alpha}$, where $H_{\alpha}$ is determined by the equation $\left(H_{\alpha}, H\right)=\alpha(H)$ 
for all $H \in \mathfrak{h}^{\mathbf{C}}$, as well as $\left[E_{\alpha}, E_{\beta}\right]=N_{\alpha, \beta} E_{\alpha+\beta}$ for $\alpha, \beta \in R, \alpha+\beta \in R$, with coefficients $N_{\alpha, \beta}$ (structural constants). These satisfy $N_{\alpha, \beta}=0$ if $\alpha+\beta \notin$ $R, \quad N_{\alpha, \beta}=-N_{\beta, \alpha}, \quad N_{-\alpha,-\beta}=-N_{\alpha, \beta}$ if $\alpha+\beta \in R$ and $N_{\alpha, \beta}=N_{\beta, \gamma}=$ $N_{\gamma, \alpha}$ if $\alpha, \beta, \gamma \in R$ and $\alpha+\beta+\gamma=0$.

The real Lie algebra $g$ is the fixed point set of the conjugation $X+i Y \mapsto$ $\overline{X+i Y}=X-i Y$ in $\mathfrak{g}^{\mathbf{C}}$. This maps $\mathfrak{g}^{(\alpha)}$ into $\mathfrak{g}^{(-\alpha)}$ [Sa, p. 185; Var, p. 289] and it is possible to choose the elements $E_{\alpha}$ such that $\overline{E_{\alpha}}=-E_{-\alpha}$ [B-F-R, p. 618]. Then $\left\{E_{\alpha}+E_{-\alpha}, i\left(E_{\alpha}-E_{-\alpha}\right)\right\}$ span $\mathfrak{g} \cap\left(\mathfrak{g}^{(\alpha)} \oplus \mathfrak{g}^{(-\alpha)}\right)$. Finally, let $\mathfrak{h}=\mathfrak{g} \cap \mathfrak{h}^{\mathbf{C}}$.

\section{2. t-ROOTS}

Having the reductive decomposition $\mathfrak{g}^{\mathrm{C}}=\mathfrak{k}^{\mathrm{C}} \oplus \mathfrak{m}^{\mathrm{C}}$ associated with the Kählerian $C$-space $M=G / K$ and the decomposition $R=R_{K} \cup R_{M}$ of the root system $R$, we set

$$
\mathfrak{t}=Z\left(\mathfrak{k}^{\mathbf{C}}\right) \cap \mathfrak{h}=\left\{X \in \mathfrak{h}: \phi(X)=0 \forall \phi \in R_{K}\right\} .
$$

(Indeed, $Z\left(\mathfrak{k}^{\mathbf{C}}\right) \cap \mathfrak{h}=\left\{X \in \mathfrak{k}^{\mathbf{C}}:[X, Y]=0\right.$ for all $\left.Y \in \mathfrak{k}^{\mathbf{C}}=\mathfrak{h} \oplus \sum_{\phi \in R_{K}} \mathbf{C} E_{\phi}\right\} \cap$ $\mathfrak{h}=\left\{X \in \mathfrak{k}^{\mathbf{C}}:\left[X, \mathfrak{h}^{\mathbf{C}}\right]=0,\left[X, E_{\phi}\right]=0 \forall \phi \in R_{K}\right\} \cap \mathfrak{h}=\left\{X \in \mathfrak{k}^{\mathbf{C}}: X \in\right.$ $\left.\left.\mathfrak{h}^{\mathbf{C}}, \phi(X) E_{\phi}=0 \forall \phi \in R_{K}\right\} \cap \mathfrak{h}=\left\{X \in \mathfrak{h}: \phi(X)=0 \forall \phi \in R_{K}\right\}\right)$.

If $\mathfrak{h}^{*}$ and $\mathfrak{t}^{*}$ are the dual spaces of $\mathfrak{h}$ and $\mathfrak{t}$, consider the restriction map

$$
\begin{aligned}
k: \mathfrak{h}^{*} & \rightarrow \mathfrak{t}^{*} \\
\alpha & \mapsto k(\alpha)=\left.\alpha\right|_{\mathfrak{t}}
\end{aligned}
$$

and set $\boldsymbol{R}_{\mathrm{t}} \equiv k(R)=k\left(\boldsymbol{R}_{M}\right)$ (note that $k\left(R_{K}\right)=0$ ). The elements of $R_{\mathrm{t}}$ are called $\mathrm{t}$-roots.

To our knowledge t-roots were first introduced in [Alek-Pe], although the above definition appears implicitly in [Sie]. The benefit from these is that there is a one-to-one correspondence between t-roots $\tau$ and irreducible $\operatorname{ad}_{\mathfrak{g}}\left(\mathrm{k}^{\mathrm{C}}\right)$ invariant submodules $M_{\tau}^{\mathrm{C}}=\sum_{k(\alpha)=\tau} \mathbf{C} E_{\alpha}$ of $\mathfrak{m}^{\mathrm{C}}$ [Sie, A]. These submodules are inequivalent, hence one has a nice description of invariant metrics and invariant complex structures on $M$ [Alek-Pe].

\section{INVARIANT METRICS}

A $G$-invariant metric on $M=G / K$ can be described by an $\operatorname{ad}_{\mathfrak{g}}(\mathfrak{k})$-invariant scalar product $g$ on $\mathfrak{m}$ [Ko-No II, p. 200]. It is also standard practice for convenience to extend $g$ without any change in notation to $\mathrm{m}^{\mathrm{C}}$. Let $\left\{\omega^{\alpha}: \alpha \in\right.$ $R\}$ be the vector space basis in $\left(\mathrm{m}^{\mathrm{C}}\right)^{*}$ which is dual to the basis $\left\{E_{\alpha}: \alpha \in\right.$ $\left.R_{M}\right\}: \omega^{\alpha}\left(E_{\beta}\right)=\delta_{\beta}^{\alpha}$. We fix a system of positive roots $R^{+}=R_{K}^{+} \cup R_{M}^{+}$where $R_{K}^{+}=R_{K} \cap R^{+}, R_{M}^{+}=R_{M} \cap R^{+}$and set $R_{\mathrm{t}}^{+}=k\left(R^{+}\right)$.

Proposition 1 [Alek]. Any real $\mathbf{a d}_{\mathfrak{g}}\left(\mathrm{k}^{\mathrm{C}}\right)$-invariant scalar product $g$ on $\mathrm{m}^{\mathrm{C}}$ has the form

$$
g=\sum_{\alpha \in R_{M}^{+}} g_{\alpha} \omega^{\alpha} \vee \omega^{-\alpha}=\sum_{\tau \in R_{t}^{+}} g_{\tau} \sum_{\alpha \in k^{-1}(\tau)} \omega^{\alpha} \vee \omega^{-\alpha}
$$

where $\omega \vee \rho=\frac{1}{2}(\omega \otimes \rho+\rho \otimes \omega), \quad g_{\alpha} \in \mathbf{R}^{+}$, and $g_{\alpha}=g_{\beta}$ if $\left.\alpha\right|_{\mathfrak{t}}=\left.\beta\right|_{\mathfrak{t}}$ so the invariant Riemannian metrics on a Kählerian $C$-space $M=G / K$ depend (modulo the scale factor) on $\left|R_{T}^{+}\right|$parameters. 


\section{The Ricci tensor AND THE EINSTEIN EQUATION}

In this section we give a detailed proof of Alekseevsky's formula $\operatorname{Ric}(X, Y)=$ $\operatorname{tr} R_{X} R_{Y}+\operatorname{tr} T_{X Y}$ [Alek] for the Ricci tensor of the invariant metric in Proposition 1 , and the corrected Lie algebraic expression for this in the case of a Kählerian $C$-space. Thus the Einstein equation reduces to an algebraic system of equations.

Let $\langle$,$\rangle be a G$-invariant Riemannian metric on $M=G / K$ and $g$ the corresponding scalar product on $\mathfrak{m}$. Denote by $\mathscr{X}(M)$ the space of all the left invariant vector fields on $M$. Recall the map

$$
\begin{aligned}
& \mathfrak{g} \rightarrow \mathscr{X}(M) \\
& X \mapsto X^{*}(p)=\left.\frac{d}{d t}(\exp t X \cdot p)\right|_{t=0}
\end{aligned}
$$

under which we got the identification $\mathfrak{m} \simeq T_{p} M$. Notice that $\left[X^{*}, Y^{*}\right]=$ $-[X, Y]^{*}$.

There is a 1-1 correspendence between $K$-invariant connections $\nabla$ on $\mathrm{M}$ and $K$-invariant linear maps $\Lambda$ (connection functions) defined by

$$
\Lambda_{X}(Y)=\left.\left(\nabla_{X^{*}} Y^{*}-L_{X^{*}} Y^{*}\right)\right|_{o}, \quad X, Y \in \mathfrak{m} .
$$

Here $o$ is the identity coset in $G / K$ and $L_{Z}$ is the Lie derivative with respect to $Z$. The linear operator $\Lambda_{X}$ is skew symmetric with respect to $g$ i.e. $g\left(\Lambda_{X} Y, Z\right)+g\left(Y, \Lambda_{X} Z\right)=0$.

The Levi-Civita connection $\nabla$ (compatible with the metric $\langle$,$\rangle ) satisfies$ the equation

$$
2\left\langle\nabla_{X^{*}} Y^{*}, Z^{*}\right\rangle=\left\langle\left[X^{*}, Y^{*}\right], Z^{*}\right\rangle+\left\langle Y^{*},\left[X^{*}, Z^{*}\right]\right\rangle+\left\langle X^{*},\left[Y^{*}, Z^{*}\right]\right\rangle
$$

from which we obtain that

$$
\left.\nabla_{X^{*}} Y^{*}\right|_{o}=-\frac{1}{2}[X, Y]_{\mathrm{m}}+U(X, Y)
$$

where the operator $U: \mathfrak{m} \times \mathfrak{m} \rightarrow \mathfrak{m}$ is determined by the condition

$$
2 g(U(X, Y), Z)=g\left([Z, X]_{\mathfrak{m}}, Y\right)+g\left(X,[Z, Y]_{\mathfrak{m}}\right) .
$$

The operator $\Lambda$ can also be written in the form:

$$
\Lambda_{X}(Y)=\frac{1}{2}[X, Y]_{\mathfrak{m}}+U(X, Y) \text {. }
$$

For all the facts above we refer to [Ko-No II, pp. 191-201], [Be, pp. 182-183], and [Gue, pp. 227-229].

For later use we need an expression for the vector $U(X, Y)$ with respect to the basis $\left\{E_{\beta}: \beta \in R_{M}\right\}$ of $\mathrm{m}^{\mathrm{c}}$. Let $U(X, Y)=\sum c_{\beta} E_{\beta}$. Then for any $\gamma \in R_{M}$

$$
2 c_{\gamma} g_{\gamma}=2 g\left(\sum c_{\beta} E_{\beta}, E_{-\gamma}\right)=g\left(\left[E_{-\gamma}, X\right]_{\mathfrak{m}}, Y\right)+g\left(X,\left[E_{-\gamma}, Y\right]_{\mathfrak{m}}\right)
$$

so

$$
U(X, Y)=\frac{1}{2} \sum_{\beta \in R_{M}} \frac{1}{g_{\beta}}\left[g\left(Y,\left[E_{-\beta}, X\right]_{\mathrm{m}}\right)+g\left(X,\left[E_{-\beta}, Y\right]_{\mathrm{m}}\right)\right] E_{\beta}
$$


In particular

$$
\begin{aligned}
U\left(E_{\alpha}, E_{\alpha}\right) & =\sum \frac{1}{g_{\beta}} g\left(E_{\alpha},\left[E_{-\beta}, E_{\alpha}\right]_{\mathfrak{m}}\right) E_{\beta} \\
& =\sum \frac{1}{g_{\beta}} N_{-\beta, \alpha} g\left(E_{\alpha}, E_{-\beta+\alpha}\right) E_{\beta}=0
\end{aligned}
$$

since $-\beta+\alpha \neq-\alpha$. By a similar argument it follows that $U\left(E_{\alpha}, E_{-\alpha}\right)=0$.

For $X, Y \in \mathfrak{m}^{\mathrm{C}}$ we define operators $R_{X}, T_{X Y}$ in $\mathrm{m}^{\mathrm{C}}$ by

$$
\begin{aligned}
& T_{X Y} Z=\frac{1}{2}\left(\left[X,[Y, Z]_{\mathfrak{k}}\right]+\left[Y,[X, Z]_{\mathfrak{k}}\right]\right), \quad Z \in \mathfrak{m}^{\mathrm{c}}, \\
& R_{X} Y=\left.\nabla_{X^{*}} Y^{*}\right|_{o} .
\end{aligned}
$$

Thus $R_{X}$ is determined by the equation

$$
-2 g\left(R_{X} Y, Z\right)=g\left(X,[Y, Z]_{\mathfrak{m}}\right)+g\left(Y,[X, Z]_{\mathfrak{m}}\right)+g\left(Z,[X, Y]_{\mathfrak{m}}\right) .
$$

Proposition 2 [Alek]. The Ricci tensor for the $\operatorname{Ad}_{G}(K)$-invariant metric $g$ is given by:

$$
\operatorname{Ric}(X, Y)=\operatorname{tr} R_{X} R_{Y}+\operatorname{tr} T_{X Y}, \quad X, Y \in \mathfrak{m}^{\mathrm{C}} .
$$

Proof. The set $\left\{F_{\alpha}\right\}=\left\{\left(E_{\alpha}+E_{-\alpha}\right) / \sqrt{2 g_{\alpha}}, i\left(E_{\alpha}-E_{-\alpha}\right) / \sqrt{2 g_{\alpha}}: \alpha \in R_{M}^{+}\right\}$ constitutes an orthonormal basis for $\mathrm{m}^{\mathrm{C}}$ with respect to $g$.

By [Wal, Lemma 7.1, p. 288] the curvature tensor $R(X, Y)$ is given by

$$
\begin{aligned}
g(R(X, Y) X, Y)= & \frac{3}{4} g\left([X, Y]_{\mathfrak{m}},[X, Y]_{\mathfrak{m}}\right)+g\left(\left[[X, Y]_{\mathfrak{k}}, Y\right], X\right) \\
& +\frac{1}{2} g\left(Y,\left[X,[X, Y]_{\mathfrak{m}}\right]_{\mathfrak{m}}\right)+\frac{1}{2} g\left(X,\left[Y,[Y, X]_{\mathfrak{m}}\right]_{\mathfrak{m}}\right) \\
& -g(U(X, Y), U(X, Y))+g(U(X, X), U(Y, Y)) .
\end{aligned}
$$

For simplicity we work with the associated quadratic forms. We compute the left-hand side.

$$
\begin{aligned}
\operatorname{Ric}(X, X)= & \sum_{\alpha \in R_{M}^{+}} g\left(R\left(X, F_{\alpha}\right) X, F_{\alpha}\right) \\
= & \frac{3}{4} \sum g\left(\left[X, F_{\alpha}\right]_{\mathfrak{m}},\left[X, F_{\alpha}\right]_{\mathfrak{m}}\right)+\sum g\left(\left[\left[X, F_{\alpha}\right]_{\mathfrak{k}}, F_{\alpha}\right], X\right) \\
& +\frac{1}{2} \sum g\left(\left[X,\left[X, F_{\alpha}\right]_{\mathfrak{m}}\right]_{\mathfrak{m}}, F_{\alpha}\right)+\frac{1}{2} \sum g\left(\left[F_{\alpha},\left[F_{\alpha}, X\right]_{\mathfrak{m}}\right]_{\mathfrak{m}}, X\right) \\
& -\sum g\left(U\left(X, F_{\alpha}\right), U\left(X, F_{\alpha}\right)+\sum g\left(U(X, X), U\left(F_{\alpha}, F_{\alpha}\right)\right)\right.
\end{aligned}
$$

For the right-hand side we need to compute $\operatorname{tr} R_{X}{ }^{2}$ and $\operatorname{tr} T_{X X}$. For the latter, using the invariance of $g$ we have

$$
\operatorname{tr} T_{X X}=\sum g\left(\left[X,\left[X, F_{\alpha}\right]_{\mathfrak{k}}, F_{\alpha}\right)=\sum g\left(X,\left[\left[X, F_{\alpha}\right]_{\mathfrak{k}}, F_{\alpha}\right]\right)\right.
$$

For $R_{X}$ we have

$$
\begin{aligned}
R_{X} & =-\frac{1}{2}[X,]_{\mathfrak{m}}+U(X, \quad) \\
& =\frac{1}{2}[X, \quad]_{\mathfrak{m}}+U(X, \quad)-[X,]_{\mathfrak{m}} \\
& =\Lambda_{X}-[X, \quad]_{\mathfrak{m}}
\end{aligned}
$$


thus

$$
\begin{aligned}
\operatorname{tr} R_{X}{ }^{2}= & \operatorname{tr} \Lambda_{X}{ }^{2}+\operatorname{tr}[X,]_{\mathfrak{m}}{ }^{2}-2 \operatorname{tr} \Lambda_{X}[X,]_{\mathfrak{m}} \\
= & -\sum g\left(\Lambda_{X} F_{\alpha}, \Lambda_{X} F_{\alpha}\right)+\sum g\left(\left[X,\left[X, F_{\alpha}\right]_{\mathfrak{m}}\right]_{\mathfrak{m}}, F_{\alpha}\right) \\
& +2 \sum g\left(\left[X, F_{\alpha}\right]_{\mathfrak{m}}, \Lambda_{X} F_{\alpha}\right) \\
= & -\sum g\left(\frac{1}{2}\left[X, F_{\alpha}\right]_{\mathfrak{m}}+U\left(X, F_{\alpha}\right), \frac{1}{2}\left[X, F_{\alpha}\right]_{\mathfrak{m}}+U\left(X, F_{\alpha}\right)\right) \\
& +\sum g\left(\left[X,\left[X, F_{\alpha}\right]_{\mathfrak{m}}\right]_{\mathfrak{m}}, F_{\alpha}\right) \\
& +2 \sum g\left(\left[X, F_{\alpha}\right]_{\mathfrak{m}}, \frac{1}{2}\left[X, F_{\alpha}\right]_{\mathfrak{m}}+U\left(X, F_{\alpha}\right)\right) \\
= & -\frac{1}{4} \sum g\left(\left[X, F_{\alpha}\right]_{\mathfrak{m}},\left[X, F_{\alpha}\right]_{\mathfrak{m}}\right)-\sum g\left(U\left(X, F_{\alpha}\right), U\left(X, F_{\alpha}\right)\right) \\
& -\sum g\left(\left[X, F_{\alpha}\right]_{\mathfrak{m}}, U\left(X, F_{\alpha}\right)\right) \\
& +\sum g\left(\left[X,\left[X, F_{\alpha}\right]_{\mathfrak{m}}\right]_{\mathfrak{m}}, F_{\alpha}\right)+\sum g\left(\left[X, F_{\alpha}\right]_{\mathfrak{m}},\left[X, F_{\alpha}\right]_{\mathfrak{m}}\right) \\
& \left.+2 \sum g\left(\left[X, F_{\alpha}\right]_{\mathfrak{m}}\right]_{\mathfrak{m}}, U\left(X, F_{\alpha}\right)\right) \\
= & \frac{3}{4} \sum g\left(\left[X, F_{\alpha}\right]_{\mathfrak{m}},\left[X, F_{\alpha}\right]_{\mathfrak{m}}\right)-\sum g\left(U\left(X, F_{\alpha}\right), U\left(X, F_{\alpha}\right)\right) \\
& +\sum g\left(\left[X, F_{\alpha}\right]_{\mathfrak{m}}, U\left(X, F_{\alpha}\right)\right)+\sum g\left(\left[X,\left[X, F_{\alpha}\right]_{\mathfrak{m}}\right]_{\mathfrak{m}}, F_{\alpha}\right) \\
= & \frac{3}{4} \sum g\left(\left[X, F_{\alpha}\right]_{\mathfrak{m}},\left[X, F_{\alpha}\right]_{\mathfrak{m}}\right)-\sum g\left(U\left(X, F_{\alpha}\right), U\left(X, F_{\alpha}\right)\right)
\end{aligned}
$$

(using the formula for $g(U(X, Y), Z)$ )

$+\frac{1}{2} \sum g\left(\left[\left[X, F_{\alpha}\right]_{\mathfrak{m}}, X\right]_{\mathfrak{m}}, F_{\alpha}\right)+\frac{1}{2} \sum g\left(X,\left[\left[X, F_{\alpha}\right]_{\mathfrak{m}}, F_{\alpha}\right]_{\mathfrak{m}}\right)$$$
+\sum g\left(\left[X,\left[X, F_{\alpha}\right]_{\mathfrak{m}}\right]_{\mathfrak{m}}, F_{\alpha}\right)
$$$$
=\frac{3}{4} \sum g\left(\left[X, F_{\alpha}\right]_{\mathfrak{m}},\left[X, F_{\alpha}\right]_{\mathfrak{m}}-\sum g\left(U\left(X, F_{\alpha}\right), U\left(X, F_{\alpha}\right)\right)\right.
$$$$
+\frac{1}{2} \sum g\left(\left[X,\left[X, F_{\alpha}\right]_{\mathfrak{m}}\right]_{\mathfrak{m}}, F_{\alpha}\right)+\frac{1}{2} \sum g\left(X,\left[\left[X, F_{\alpha}\right]_{\mathfrak{m}}, F_{\alpha}\right]_{\mathfrak{m}}\right) \text {. }
$$

If we sum $\operatorname{tr} R_{X}^{2}$ and $\operatorname{tr} T_{X X}$ we get the left-hand side, and the proof will have been completed, provided we show that $\sum g\left(U(X, X), U\left(F_{\alpha}, F_{\alpha}\right)\right)=0$.

Now, $U\left(F_{\alpha}, F_{\alpha}\right)=0$ since this reduces to computing $U\left(E_{\alpha}, E_{\alpha}\right)$ and $U\left(E_{\alpha}, E_{-\alpha}\right)$, which are zero as shown before.

The Ricci tensor is now determined by its value on the basis $\left\{E_{\alpha}: \alpha \in R_{M}\right\}$.

Proposition 3. The Ricci tensor for an invariant Riemannian metric $g$ described in the previous section is given by

$$
\operatorname{Ric}\left(E_{\alpha}, E_{\beta}\right)=0, \quad \alpha, \beta \in R_{M}, \alpha+\beta \notin R_{M},
$$

$$
\operatorname{Ric}\left(E_{\alpha}, E_{-\alpha}\right)=(\alpha, \alpha)+\sum_{\substack{\phi \in R_{K} \\ \alpha+\phi \in R}} N_{\alpha, \phi}^{2}+\frac{1}{4} \sum_{\beta \in R_{M}^{*}} \frac{N_{\alpha, \beta}^{2}}{g_{\alpha+\beta} g_{\beta}}\left(g_{\alpha}^{2}-\left(g_{\alpha+\beta}-g_{\beta}\right)^{2}\right)
$$

where $R_{M}^{*}=R_{M}-k^{-1}(k(\alpha))$. 
Remarks. (a) The restriction in the second sum above, comes from the fact that if $\beta \in k^{-1}(k(\alpha))$, then $\alpha+\beta \notin R_{M}$. (Indeed, if $\alpha+\beta \in R_{M}$, then $k(\alpha+\beta) \in R_{\mathrm{t}}$; but $k(\alpha+\beta)=k(\alpha)+k(\beta)=2 k(\alpha)$ which is not a $\mathrm{t}$-root.)

(b) The Einstein equation $\operatorname{Ric}(g)=c g$ reduces to an algebraic system of $\left|R_{T}^{+}\right|$equations with $\left|R_{T}^{+}\right|+1$ unknowns, namely $g_{\tau}\left(\tau \in R_{T}^{+}\right)$and $c$. Since $\operatorname{Ric}(\lambda g)=\operatorname{Ric}(g)$ we can normalize either one of the $g_{\alpha}$ or $c$ to 1 , so essentially we get $\left|R_{T}^{+}\right|$equations with $\left|R_{T}^{+}\right|$unknowns.

Proof. The first part is direct from the relation $\left[E_{\alpha}, E_{\beta}\right]=0$ if $\alpha+\beta \notin R_{M}$, Proposition 2 and the definitions of $T_{X Y}$ and $R_{X}$.

For the second, fix an $E_{\alpha}$ in $\mathfrak{m}^{\mathrm{C}}$. Then for any operator $\mathrm{T}$ on $\mathfrak{m}^{\mathrm{C}}$ (using the orthonormal basis $\left\{F_{\alpha}\right\}$ )

$$
\operatorname{tr} T=\sum_{\alpha \in R_{M}} \frac{1}{g_{\alpha}} g\left(T\left(E_{\alpha}\right), E_{-\alpha}\right) .
$$

By Proposition 2, we need to compute $\operatorname{tr} T_{E_{\alpha} E_{-\alpha}}$ and $\operatorname{tr} R_{E_{\alpha}} R_{E_{-\alpha}}$. We have

$$
\begin{aligned}
\operatorname{tr} T_{E_{\alpha} E_{-\alpha}}= & \sum_{\beta \in R_{M}} \frac{1}{g_{\beta}} g\left(T_{E_{\alpha} E_{-\alpha}}\left(E_{\beta}\right), E_{-\beta}\right) \\
= & \frac{1}{2} \sum \frac{1}{g_{\beta}} g\left(\left[E_{\alpha},\left[E_{-\alpha}, E_{\beta}\right]_{\mathrm{\ell}}\right], E_{-\beta}\right) \\
& +\frac{1}{2} \sum \frac{1}{g_{\beta}} g\left(\left[E_{-\alpha},\left[E_{\alpha}, E_{\beta}\right]_{\mathrm{\ell}}\right], E_{-\beta}\right) \\
= & \frac{1}{2} \frac{1}{g_{\alpha}} g\left(\left[E_{\alpha},\left[E_{-\alpha}, E_{\alpha}\right]_{\mathrm{\ell}}\right], E_{-\alpha}\right)+\frac{1}{2} \sum_{\beta \neq \alpha} \frac{1}{g_{\beta}} g\left(\left[E_{\alpha},\left[E_{-\alpha}, E_{\beta}\right]_{\mathrm{\ell}}\right], E_{-\beta}\right) \\
& +\frac{1}{2} \frac{1}{g_{\alpha}} g\left(\left[E_{-\alpha},\left[E_{\alpha}, E_{-\alpha}\right]_{\mathrm{\ell}}\right], E_{\alpha}\right) \\
& +\frac{1}{2} \sum_{\beta \neq-\alpha} \frac{1}{g_{\beta}} g\left(\left[E_{-\alpha},\left[E_{\alpha}, E_{\beta}\right]_{\mathrm{\ell}}\right], E_{-\beta}\right) \\
= & \frac{1}{2} \frac{1}{g_{\alpha}} g\left(\left[H_{\alpha}, E_{\alpha}\right], E_{-\alpha}\right)+\frac{1}{2} \sum_{\beta \neq \alpha} \frac{1}{g_{\beta}} g\left(\left[E_{\alpha}, N_{-\alpha, \beta} E_{-\alpha+\beta}\right], E_{-\beta}\right) \\
& +\frac{1}{2} \frac{1}{g_{\alpha}} g\left(-\left[H_{\alpha}, E_{-\alpha}\right], E_{\alpha}\right)+\frac{1}{2} \sum_{\beta \neq-\alpha} \frac{1}{g_{\beta}} g\left(\left[E_{-\alpha}, N_{\alpha, \beta} E_{\alpha+\beta}\right], E_{-\beta}\right) \\
= & \frac{1}{2} \frac{1}{g_{\alpha}} g\left(\alpha\left(H_{\alpha}\right) E_{\alpha}, E_{-\alpha}\right)+\frac{1}{2} \sum_{\phi \in R_{K}} \frac{1}{g_{\alpha+\phi}} g\left(\left[E_{\alpha}, N_{-\alpha, \alpha+\phi} E_{\phi}\right], E_{-(\alpha+\phi)}\right) \\
& +\frac{1}{2} \frac{1}{g_{\alpha}} g\left(\alpha\left(H_{\alpha}\right) E_{-\alpha}, E_{\alpha}\right) \\
& +\frac{1}{2} \sum_{\psi \in R_{K}} \frac{1}{g_{\alpha+\psi}} g\left(\left[E_{-\alpha}, N_{\alpha,-(\alpha+\psi)} E_{-\psi}\right], E_{\alpha+\psi}\right)
\end{aligned}
$$




$$
\begin{aligned}
= & \frac{(\alpha, \alpha)}{2}+\frac{(\alpha, \alpha)}{2}+\frac{1}{2} \sum_{\phi} \frac{1}{g_{\alpha+\phi}} N_{-\alpha, \alpha+\phi} N_{\alpha, \phi} g\left(E_{\alpha+\phi}, E_{-(\alpha+\phi)}\right) \\
& +\frac{1}{2} \sum_{\psi} \frac{1}{g_{\alpha+\psi}} N_{\alpha,-(\alpha+\psi)} N_{-\alpha,-\psi} g\left(E_{-(\alpha+\psi)}, E_{\alpha+\psi}\right) \\
= & (\alpha, \alpha)+\sum_{\substack{\phi \in R_{K} \\
\alpha+\phi \in R}} N_{-\alpha, \alpha+\phi} N_{\alpha, \phi}=(\alpha, \alpha)+\sum_{\phi \in R_{K}} N_{\alpha, \phi}^{2} .
\end{aligned}
$$

We now come to $\operatorname{tr} R_{E_{\alpha}} R_{E_{-\alpha}}$. Using a similar argument as for $U(X, Y)$ we get that

$$
\begin{aligned}
R_{X} Y=-\frac{1}{2} \sum_{\gamma \in R_{M}} \frac{1}{g_{\gamma}}\left[g\left([X, Y]_{\mathfrak{m}}, E_{-\gamma}\right)+g\left(X,\left[Y, E_{-\gamma}\right]_{\mathfrak{m}}\right)\right. & \\
& \left.+g\left(Y,\left[X, E_{-\gamma}\right]_{\mathfrak{m}}\right)\right] E_{\gamma} .
\end{aligned}
$$

Replacing $X$ by $Z, Y$ by $E_{\gamma}$, and $E_{\gamma}$ by $E_{\delta}$, gives

$$
\begin{aligned}
R_{Z} E_{\gamma}=-\frac{1}{2} \sum_{\delta \in R_{M}} \frac{1}{g_{\delta}}\left[g\left(\left[Z, E_{\gamma}\right]_{\mathfrak{m}}, E_{-\delta}\right)+g\left(Z,\left[E_{\gamma}, E_{-\delta}\right]_{\mathfrak{m}}\right)\right. & \\
& \left.+g\left(E_{\gamma},\left[Z, E_{-\delta}\right]_{\mathfrak{m}}\right)\right] E_{\delta}
\end{aligned}
$$

Applying $R_{Z}$ to $R_{X} Y$ we get

$$
\begin{aligned}
R_{Z} R_{X} Y= & \frac{1}{4} \sum_{\gamma, \delta \in R_{M}} \frac{1}{g_{\gamma} g_{\delta}}\left[g\left([X, Y]_{\mathfrak{m}}, E_{-\gamma}\right)+g\left(X,\left[Y, E_{-\gamma}\right]_{\mathfrak{m}}\right)\right. \\
& \left.+g\left(Y,\left[X, E_{-\gamma}\right]_{\mathfrak{m}}\right)\right] \\
& \times\left[g\left(\left[Z, E_{\gamma}\right]_{\mathfrak{m}}, E_{-\delta}\right)+g\left(Z,\left[E_{\gamma}, E_{-\delta}\right]_{\mathfrak{m}}\right)+g\left(E_{\gamma},\left[Z, E_{-\delta}\right]_{\mathfrak{m}}\right)\right] E_{\delta} .
\end{aligned}
$$

Thus

$$
\begin{aligned}
\operatorname{tr} R_{E_{\alpha}} & R_{E_{-\alpha}}=\sum_{\beta \in R_{M}} \frac{1}{g_{\beta}} g\left(R_{E_{\alpha}} R_{E_{-\alpha}}\left(E_{\beta}\right), E_{-\beta}\right) \\
= & \frac{1}{4} \sum_{\beta, \gamma, \delta} \frac{1}{g_{\beta} g_{\gamma} g_{\delta}}\left\{g\left(\left[E_{-\alpha}, E_{\beta}\right]_{\mathfrak{m}}, E_{-\gamma}\right)+g\left(E_{-\alpha},\left[E_{\beta}, E_{-\gamma}\right]_{\mathfrak{m}}\right)\right. \\
& \left.\times\left\{g\left(\left[E_{\alpha}, E_{\gamma}\right]_{\mathfrak{m}}, E_{-\delta}\right)+g\left(E_{\alpha},\left[E_{\gamma}, E_{-\delta}\right]_{\mathfrak{m}}\right) \quad+g\left(E_{-\alpha}, E_{-\gamma}\right]_{\mathfrak{m}}\right)\right\} \\
\quad & \left.\left.\quad+\left[E_{\alpha}, E_{-\delta}\right]_{\mathfrak{m}}\right)\right\} g\left(E_{\delta}, E_{-\beta}\right) \\
= & \begin{array}{c}
\frac{1}{4} \sum_{\beta, \gamma} \frac{1}{g_{\beta} g_{\gamma}}\left\{N_{-\alpha, \beta} g\left(E_{-\alpha+\beta}, E_{-\gamma}\right)+N_{\beta,-\gamma} g\left(E_{-\alpha}, E_{\beta-\gamma}\right)\right. \\
\left.\quad+N_{-\alpha,-\gamma} g\left(E_{\beta}, E_{-\alpha-\gamma}\right)\right\}
\end{array} \\
& \times\left\{N_{\alpha, \gamma} g\left(E_{\alpha+\gamma}, E_{-\beta}\right)+N_{\gamma,-\beta} g\left(E_{\alpha}, E_{\gamma-\beta}\right)+N_{\alpha,-\beta} g\left(E_{\gamma}, E_{\alpha-\beta}\right)\right\}
\end{aligned}
$$




$$
\begin{aligned}
&=\frac{1}{4} \sum_{\gamma} \frac{1}{g_{\alpha+\gamma} g_{\gamma}}\left(N_{-\alpha, \alpha+\gamma} g_{\gamma}+\right.\left.N_{\alpha+\gamma,-\gamma} g_{\alpha}+N_{-\alpha,-\gamma} g_{\alpha+\gamma}\right) \\
& \quad \times\left(N_{\alpha, \gamma} g_{\alpha+\gamma}+N_{\gamma,-(\alpha+\gamma)} g_{\alpha}+N_{\alpha,-(\alpha+\gamma)} g_{\gamma}\right) \\
&=\frac{1}{4} \sum_{\beta} \frac{1}{g_{\alpha+\beta} g_{\beta}}\left(N_{\beta, \alpha} g_{\alpha+\beta}-\right.\left.N_{\beta,-(\alpha+\beta)} g_{\alpha}-N_{\alpha,-(\alpha+\beta)} g_{\beta}\right) \\
& \quad \times\left(N_{\alpha+\beta,-\alpha} g_{\beta}-N_{\alpha+\beta,-\beta} g_{\alpha}-N_{-\alpha,-\beta} g_{\alpha+\beta}\right) \\
&=\frac{1}{4} \sum_{\beta} \frac{1}{g_{\alpha+\beta}, g_{\beta}}\left(-N_{\alpha, \beta} g_{\alpha+\beta}-N_{\alpha, \beta} g_{\alpha}+N_{\alpha, \beta} g_{\beta}\right) \quad \times\left(N_{-\alpha-\beta} g_{\beta}+N_{-\alpha,-\beta} g_{\alpha}-N_{-\alpha,-\beta} g_{\alpha+\beta}\right) \\
&=\frac{1}{4} \sum_{\beta} \frac{-N_{\alpha, \beta} N_{-\alpha,-\beta}}{g_{\alpha+\beta} g_{\beta}}\left(g_{\alpha}+g_{\alpha+\beta}-g_{\beta}\right)\left(g_{\alpha}-g_{\alpha+\beta}+g_{\beta}\right) \\
&=\frac{1}{4} \sum_{\beta} \frac{N_{\alpha, \beta}^{2}}{g_{\alpha+\beta} g_{\beta}}\left(g_{\alpha}^{2}-\left(g_{\alpha+\beta}-g_{\beta}\right)^{2}\right) .
\end{aligned}
$$

\section{SOlutions OF THE EINSTEIN EQUATION FOR CERTAIN KÄHLERIAN $C$-SPACES}

In this section we present the Einstein equation for certain Kählerian $C$ spaces and in some cases we give explicit solutions. Here we would like to mention that in the process of solving the systems of the Einstein equations to be presented below, we include the Einstein constant in the equations, but then rescale in order to present the solutions (up to scale) in a simple form.

$$
\text { (A) The space } S U(n) / S\left(U\left(n_{1}\right) \times \cdots \times U\left(n_{s}\right)\right), \quad n=\sum_{i=1}^{s} n_{i} \text {. }
$$

A Cartan subalgebra for the complexification of the Lie algebra su(n) consists of the diagonal matrices

$$
\mathfrak{h}^{\mathbf{C}}=\left\{\operatorname{diag}\left(\varepsilon_{1}, \varepsilon_{2}, \ldots, \varepsilon_{n}\right) \quad \varepsilon_{i} \in \mathbf{C}, \quad \sum_{i=1}^{n} \varepsilon_{i}=0\right\} .
$$

The root system has the form $R=\left\{\varepsilon_{i}-\varepsilon_{j}: i \neq j\right\}$ and the positive roots may be chosen as $R^{+}=\left\{\varepsilon_{i}-\varepsilon_{j}: i<j\right\}$. For convenience we set

$$
\varepsilon_{a}^{i}=\varepsilon_{n_{1}+\cdots+n_{i-1}+a}, \quad i=1, \ldots, s, \quad a=1, \ldots, n_{i} .
$$

Then the root system for the complexification of the subalgebra denoted by $\mathfrak{k}=\mathbf{s}\left(\mathbf{u}\left(n_{1}\right) \times \cdots \times \mathbf{u}\left(n_{s}\right)\right)$ is $R_{K}=\left\{\varepsilon_{a}^{i}-\varepsilon_{b}^{i}: 1 \leq a \neq b \leq n_{i}\right\}$. So we get $R^{+}=\left\{\varepsilon_{a}^{i}-\varepsilon_{b}^{i}: 1 \leq a<b \leq n_{i} ; \varepsilon_{a}^{i}-\varepsilon_{b}^{j}: i<j\right\}, \quad R_{K}^{+}=\left\{\varepsilon_{a}^{i}-\varepsilon_{b}^{i}: 1 \leq a<b \leq\right.$ $\left.n_{i}\right\}, \quad R_{M}^{+}=\left\{\varepsilon_{a}^{i}-\varepsilon_{b}^{j}: i<j\right\}$.

The center of $\mathfrak{k}$ as a subalgebra of $\mathfrak{h}^{\mathrm{C}}$ is of the form

$$
\operatorname{diag}\left(\varepsilon^{1}, \ldots, \varepsilon^{1}, \varepsilon^{2}, \ldots, \varepsilon^{2}, \ldots, \varepsilon^{s}, \ldots, \varepsilon^{s}\right)
$$

(where $\varepsilon^{i}$ appears $n_{i}$ times, $i=1, \ldots, s$ ) hence the number of the positive 
t-roots (by restricting the roots $\left.\varepsilon_{a}^{i}-\varepsilon_{b}^{j}\right)$ is $\frac{1}{2} s(s-1)$.

The Killing form for $S U(n)$ is $(X, Y)=2 n \operatorname{tr} X Y$, we have $(\alpha, \alpha)=1 / n$ for all roots $\alpha$, and root vectors $E_{\alpha}$ that satisfy $\left(E_{\alpha}, E_{-\alpha}\right)=-1$ are of the form $E_{a b}^{i j}=E_{\varepsilon_{a}^{i}-\varepsilon_{b}^{j}} / \sqrt{2 n}$ where $E_{\varepsilon_{a}^{i}-\varepsilon_{b}^{j}}$ is the root vector corresponding to the root $\varepsilon_{a}^{i}-\varepsilon_{b}^{j}$. The structural constants are all equal to $1 / \sqrt{2 n}$. From Proposition 3 we get

$$
\operatorname{Ric}\left(E_{a b}^{i j}, E_{b a}^{j i}\right)=\frac{1}{n}+\frac{n_{i}+n_{j}-2}{2 n}+\frac{1}{4 n} \sum_{l \neq i, j} \frac{n_{l}}{g_{i l} g_{j l}}\left(g_{i j}^{2}-\left(g_{i l}-g_{j l}\right)^{2}\right)
$$

where $g_{i j}=g\left(E_{a b}^{i j}, E_{b a}^{j i}\right), 1 \leq i<j \leq n$.

Proposition 4 [Alek]. For the space $S U(n) / S\left(U\left(n_{1}\right) \times \cdots \times U\left(n_{s}\right)\right)$ the Einstein equation reduces to the system

$$
n_{i}+n_{j}+\frac{1}{2} \sum_{l \neq i, j} \frac{n_{l}}{g_{i l} g_{j l}}\left(g_{i j}^{2}-\left(g_{i l}-g_{j l}\right)^{2}\right)=g_{i j}
$$

of $\frac{1}{2} s(s-1)$ equations with $\frac{1}{2} s(s-1)$ unknowns $g_{i j}$.

For $s=3$ we get

Theorem 5. The space $S U(n) / S\left(U\left(n_{1}\right) \times U\left(n_{2}\right) \times U\left(n_{3}\right)\right)$ admits (up to scale) precisely four invariant Einstein metrics:

(a) $g_{12}=n_{1}+n_{2}, \quad g_{13}=n_{1}+2 n_{2}+n_{3}, \quad g_{23}=n_{2}+n_{3}$,

(b) $g_{12}=n_{1}+n_{2}+2 n_{3}, \quad g_{13}=n_{1}+n_{3}, \quad g_{23}=n_{2}+n_{3}$,

(c) $g_{12}=n_{1}+n_{2}, \quad g_{13}=n_{1}+n_{3}, \quad g_{23}=2 n_{1}+n_{2}+n_{3}$,

(d) $g_{12}=n_{1}+n_{2}, \quad g_{13}=n_{1}+n_{3}, \quad g_{23}=n_{2}+n_{3}$.

The solutions (a)-(c) are Kähler metrics and (d) is non-Kähler. Notice that if $n_{1}=n_{2}=n_{3}$ then $(\mathrm{d})$ is Wang-Ziller's normal Einstein metric.

(B) The space $S U(n) / S(U(1) \times \cdots \times U(1))$ (n factors equal to $U(1))$.

Everything said in case (A) applies here so we only set $E^{i j}=E_{\varepsilon_{i}-\varepsilon_{j}}$ and $n_{i}=$ $n_{j}=1$ in $(*)$ to obtain

Proposition 6. For the space $S U(n) / S(U(1) \times \cdots \times U(1))$ the Einstein equation reduces to the system

$$
2+\frac{1}{2} \sum_{l \neq i, j} \frac{1}{g_{i l} g_{j l}}\left(g_{i j}^{2}-\left(g_{i l}-g_{j l}\right)^{2}\right)=g_{i j}
$$

of $\frac{1}{2} n(n-1)$ equations with $\frac{1}{2} n(n-1)$ unknowns $g_{i j}$.

It is known [W-Z] that besides the Kähler-Einstein metrics the above spaces admit the normal metric on $M=G / K$ (this is the canonical metric on a 
homogeneous space which is induced by minus the Killing form of $\mathfrak{g}$ restricted to $\mathfrak{m})$ as Einstein. This is given by setting all the numbers $g_{i j}$ equal. The exact number of Einstein metrics for any $n$ is not known in general though. We only get the following result:

Theorem 7. The space $S U(n) / S(U(1) \times \cdots \times U(1)$ ) for $n=3$ admits (up to scale) the normal and the Kähler-Einstein metrics and for $n \geq 4$ admits at least $n ! / 2+1+n$ Einstein metrics. The $n ! / 2$ metrics are Kähler-Einstein, one is the normal metric and the remaining $n$ are given explicitly as follows:

$$
\begin{aligned}
& g_{s i}=g_{s j}=n-1, \quad i \neq s, \quad j \neq s, \\
& g_{k l}=n+1, \quad k, l \neq s(1 \leq s \leq n) .
\end{aligned}
$$

(C) The space $S O(2 n) / U\left(n_{1}\right) \times \cdots \times U\left(n_{s}\right), \quad n=\sum_{i=1}^{s} n_{i}$.

A Cartan subalgebra for the complexification of the Lie algebra so $(2 n)$ is of the form

$$
\mathfrak{h}^{\mathbf{C}}=\left\{\operatorname{diag}\left(\varepsilon_{1},-\varepsilon_{1}, \ldots, \varepsilon_{n},-\varepsilon_{n}\right) \varepsilon_{i} \in \mathbf{C}\right\} .
$$

The root system has the form

$$
R=\left\{ \pm\left(\varepsilon_{i}-\varepsilon_{j}\right): 1 \leq i<j \leq n\right\} \cup\left\{ \pm\left(\varepsilon_{i}+\varepsilon_{j}\right): 1 \leq i<j \leq n\right\}
$$

and the positive roots may be chosen as the forms $R^{+}=\left\{\varepsilon_{i}-\varepsilon_{j}: 1 \leq i<\right.$ $j \leq n\} \cup\left\{\varepsilon_{i}+\varepsilon_{j}: 1 \leq i<j \leq n\right\}$. The root system for the subalgebra $\mathfrak{k}^{\mathrm{C}}=\mathbf{u}\left(n_{1}\right) \times \cdots \times \mathbf{u}\left(n_{s}\right)$ is $R_{K}=\left\{\varepsilon_{c}^{i}-\varepsilon_{d}^{i}: 1 \leq c \neq d \leq n_{i}\right\}$, thus we have $R_{M}^{+}=$ $\left\{\varepsilon_{a}^{i}-\varepsilon_{b}^{j}, \varepsilon_{a}^{i}+\varepsilon_{b}^{j}: i<j, \varepsilon_{a}^{i}+\varepsilon_{b}^{i}: a<b\right\}$ and $R_{K}^{+}=\left\{\varepsilon_{c}^{i}-\varepsilon_{d}^{i}: 1 \leq c<d \leq n_{i}\right\}$.

The center of $\mathfrak{k}^{\mathbf{C}}$ as a subalgebra of $\mathfrak{h}^{\mathrm{C}}$ has the form

$$
\operatorname{diag}\left(\varepsilon^{1},-\varepsilon^{1}, \ldots, \varepsilon^{1},-\varepsilon^{1}, \ldots, \varepsilon^{s},-\varepsilon^{s}, \ldots, \varepsilon^{s},-\varepsilon^{s}\right), \quad \varepsilon^{i} \in \mathbf{C},
$$

(where $\varepsilon^{i}$ appears $n_{i}$ times, $i=1, \ldots, s$ ) hence by restricting the roots $\varepsilon_{a}^{i}-\varepsilon_{b}^{j}$ $(i<j), \quad \varepsilon_{a}^{i}+\varepsilon_{b}^{j} \quad(i<j), \quad \varepsilon_{a}^{i}+\varepsilon_{b}^{i} \quad(a<b)$ in $Z\left(\mathfrak{k}^{\mathbf{C}}\right)$ we get $\frac{1}{2} s(s-1)+$ $\frac{1}{2} s(s-1)+s=s^{2}$ positive t-roots.

The Killing form for $S O(2 n)$ is $(X, Y)=2(n-1) \operatorname{tr} X Y,(\alpha, \alpha)=\frac{1}{2}(n-1)$ for all roots $\alpha$, and root vectors $E_{\alpha}$ satisfying $\left(E_{\alpha}, E_{-\alpha}\right)=-1$ are $E_{a b}^{i j}=$ $\left(\frac{1}{2} \sqrt{n-1}\right) E_{\varepsilon_{a}^{i}-\varepsilon_{b}^{j}}, \quad F_{a b}^{i j}=\left(\frac{1}{2} \sqrt{n-1}\right) E_{\varepsilon_{a}^{i}+\varepsilon_{b}^{j}}, G_{a b}^{i}=\left(\frac{1}{2} \sqrt{n-1}\right) E_{\varepsilon_{a}^{i}+\varepsilon_{b}^{i}}$; the structural constants have absolute value $\frac{1}{2} \sqrt{n-1}$. The calculation of the Ricci tensor is more laborious in this example due to the three "kinds" of roots. We obtain:

$$
\begin{aligned}
\operatorname{Ric}\left(E_{a b}^{i j}, E_{b a}^{j i}\right)= & \frac{1}{2(n-1)}+\frac{n_{i}+n_{j}-2}{4(n-1)}+\frac{1}{8(n-1)} \sum_{l \neq i, j} \frac{n_{l}}{g_{i l} g_{j l}}\left(g_{i j}^{2}-\left(g_{i l}-g_{j l}\right)^{2}\right) \\
& +\frac{1}{8(n-1)} \sum_{l \neq i, j} \frac{n_{l}}{f_{i l} f_{j l}}\left(g_{i j}^{2}-\left(f_{i l}-f_{j l}\right)^{2}\right) \\
& +\frac{1}{8(n-1)} \frac{n_{i}-1}{f_{i j} h_{i}}\left(g_{i j}^{2}-\left(f_{i j}^{2}-h_{i}\right)^{2}\right) \\
& +\frac{1}{8(n-1)} \frac{n_{j}-1}{f_{i j} h_{j}}\left(g_{i j}^{2}-\left(f_{i j}-h_{j}\right)^{2}\right),
\end{aligned}
$$




$$
\begin{aligned}
\operatorname{Ric}\left(F_{a b}^{i j}, F_{b a}^{j i}\right)= & \frac{1}{2(n-1)}+\frac{n_{i}+n_{j}-2}{4(n-1)}+\frac{1}{8(n-1)} \sum_{l \neq i, j} \frac{n_{l}}{g_{i l} g_{j l}}\left(f_{i j}^{2}-\left(g_{i l}-g_{j l}\right)^{2}\right) \\
& +\frac{1}{8(n-1)} \sum_{l \neq i, j} \frac{n_{l}}{f_{i l} g_{j l}}\left(f_{i j}^{2}-\left(f_{i l}-g_{j l}\right)^{2}\right) \\
& +\frac{1}{8(n-1)} \frac{n_{i}-1}{g_{i j} h_{i}}\left(f_{i j}^{2}-\left(g_{i j}^{2}-h_{i}\right)^{2}\right) \\
& +\frac{1}{8(n-1)} \frac{n_{i}-1}{g_{i j} h_{j}}\left(f_{i j}^{2}-\left(g_{i j}-h_{j}\right)^{2}\right),
\end{aligned}
$$

$\operatorname{Ric}\left(G_{a b}^{i}, G_{b a}^{i}\right)=\frac{1}{2(n-1)}+\frac{n_{i}-2}{2(n-1)}+\frac{1}{4(n-1)} \sum_{l \neq i} \frac{n_{l}}{g_{i l} f_{i l}}\left(h_{i}^{2}-\left(g_{i l}-f_{i l}\right)^{2}\right)$

where $g_{i j}=g\left(E_{a b}^{i j}, E_{b a}^{j i}\right), \quad f_{i j}=g\left(F_{a b}^{i j}, F_{b a}^{j i}\right) \quad(1 \leq i<j \leq s), \quad h_{i}=$ $g\left(G_{a b}^{i}, G_{b a}^{i}\right), \quad 1 \leq i \leq s$. We get

Proposition 8. For the space $S O(2 n) / U\left(n_{1}\right) \times \cdots \times U\left(n_{s}\right)$ the Einstein equation reduces to the following system of $s^{2}$ equations with $s^{2}$ unknowns $g_{i j}, f_{i j}, h_{i}$ :

$$
\begin{gathered}
n_{i}+n_{j}+\frac{1}{2}\left\{\sum_{l \neq i, j} \frac{n_{l}}{g_{i l} g_{j l}}\left(g_{i j}^{2}-\left(g_{i l}-g_{j l}\right)^{2}\right)+\sum_{l \neq i, j} \frac{n_{l}}{f_{i l} f_{j l}}\left(g_{i j}^{2}-\left(f_{i l}-f_{j l}\right)^{2}\right)\right. \\
\left.+\frac{n_{i}-1}{f_{i j} h_{i}}\left(g_{i j}^{2}-\left(f_{i j}-h_{i}\right)^{2}\right)+\frac{n_{j}-1}{f_{i j} h_{j}}\left(g_{i j}^{2}-\left(f_{i j}-h_{j}\right)^{2}\right)\right\}=g_{i j}, \\
n_{i}+n_{j}+\frac{1}{2}\left\{\sum_{l \neq i, j} \frac{n_{l}}{g_{i l} g_{j l}}\left(f_{i j}^{2}-\left(g_{i l}-f_{j l}\right)^{2}\right)+\sum_{l \neq i, j} \frac{n_{l}}{f_{i l} g_{j l}}\left(f_{i j}^{2}-\left(f_{i l}-g_{j l}\right)^{2}\right)\right. \\
\left.+\frac{n_{i}-1}{g_{i j} h_{i}}\left(f_{i j}^{2}-\left(g_{i j}-h_{i}\right)^{2}\right)+\frac{n_{j}-1}{g_{i j} h_{j}}\left(f_{i j}^{2}-\left(g_{i j}-h_{j}\right)^{2}\right)\right\}=f_{i j}, \\
2\left(n_{i}-1\right)+\sum_{l \neq i} \frac{n_{l}}{g_{i l} f_{i l}}\left(h_{i}^{2}-\left(g_{i l}-f_{i l}\right)^{2}\right)=h_{i} .
\end{gathered}
$$

For $s=2$ and $n_{1}=n_{2}=m$ the system has four unknowns $f, g, h_{1}, h_{2}$.

Theorem 9. The space $S O(2 n) / U(m) \times U(m)$ (where $n=2 m$ ) admits (up to scale) precisely ten invariant Einstein metrics, explicitly given as follows:

$$
\begin{aligned}
\text { (a)-(b) } h_{1} & =h_{2}=1, f=g=\frac{2 m-1 \pm \sqrt{2 m-1}}{2(m-1)} \\
\text { (c)-(d) } h_{2} & =\frac{7 m^{3}-m^{2}-3 m+1 \pm 2(2 m-1) \sqrt{2 m\left(-m^{3}+7 m^{2}-5 m+1\right)}}{(m-1)(3 m-1)^{2}}, \\
g & =\sqrt{\frac{m}{2(m-1)} h_{2}}, f=g, h_{1}=1,
\end{aligned}
$$


(e) - (f) $h_{1}=\frac{7 m^{3}-m^{2}-3 m+1 \pm 2(2 m-1) \sqrt{2 m\left(-m^{3}+7 m^{2}-5 m+1\right)}}{(m-1)(3 m-1)^{2}}$, $g=\sqrt{\frac{m}{2(m-1)} h_{1}}, f=g, h_{2}=1$,

(g) $g=m, h_{1}=(m-1), f=(2 m-1), h_{2}=(3 m-1)$,

(h) $g=m, h_{1}=(3 m-1), f=(2 m-1), h_{2}=(m-1)$,

(i) $g=(2 m-1), h_{1}=(3 m-1), f=m, h_{2}=(m-1)$,

(j) $g=(2 m-1), h_{1}=(m-1), f=m, h_{2}=(3 m-1)$.

The metrics $(\mathrm{g}),(\mathrm{h}),(\mathrm{i})$ and $(\mathrm{j})$ are Kähler and the remaining six are non-Kähler.

\section{(D) The space $G_{2} / U(2)$}

A Cartan subalgebra of the complexification of the Lie algebra $\mathbf{G}_{2}$ consists of matrices of the form

$$
\mathfrak{h}^{\mathbf{C}}=\left\{\operatorname{diag}\left(0, \varepsilon_{1}, \varepsilon_{2}, \varepsilon_{3},-\varepsilon_{1},-\varepsilon_{2},-\varepsilon_{3}\right), \varepsilon_{i} \in \mathbf{C}, \sum \varepsilon_{i}=0\right\}
$$

(usually viewed as a subalgebra of so(7)).

The root system has the form $R=\left\{ \pm \varepsilon_{1}, \pm \varepsilon_{2}, \mp\left(\varepsilon_{1}+\varepsilon_{2}\right), \pm\left(\varepsilon_{1}-\varepsilon_{2}\right)\right.$, $\left.\pm\left(2 \varepsilon_{1}+\varepsilon_{2}\right), \pm\left(\varepsilon_{1}+2 \varepsilon_{2}\right)\right\}$, and $R_{K}^{+}=\left\{\varepsilon_{1}-\varepsilon_{2}\right\}, R_{M}^{+}=\left\{\varepsilon_{1}, \varepsilon_{2},-\left(\varepsilon_{1}+\varepsilon_{2}\right),\left(2 \varepsilon_{1}+\right.\right.$ $\left.\left.\varepsilon_{2}\right),\left(\varepsilon_{1}+2 \varepsilon_{2}\right)\right\}$. Here $\mathbf{k}=\mathbf{u}(2)$ and its center as a subalgebra of $\mathfrak{h}^{\mathbf{C}}$ has the form

$$
\operatorname{diag}(0, \varepsilon, \varepsilon,-2 \varepsilon,-\varepsilon,-\varepsilon, 2 \varepsilon) \text {. }
$$

We get three positive $t$-roots, thus three unknowns for the Einstein equation:

$$
\begin{aligned}
& g_{1}=g\left(E_{\varepsilon_{1}}, E_{-\varepsilon_{1}}\right)=g\left(E_{\varepsilon_{2}}, E_{-\varepsilon_{2}}\right), \quad g_{2}=g\left(E_{-\left(\varepsilon_{1}+\varepsilon_{2}\right)}, E_{\left(\varepsilon_{1}+\varepsilon_{2}\right)}\right), \\
& g_{3}=g\left(E_{2 \varepsilon_{1}+\varepsilon_{2}}, E_{-\left(2 \varepsilon_{1}+\varepsilon_{2}\right)}\right)=g\left(E_{\varepsilon_{1}+2 \varepsilon_{2}}, E_{-\left(\varepsilon_{1}+2 \varepsilon_{2}\right)}\right) .
\end{aligned}
$$

In this example there are "long" and "short" roots $\alpha$ and $\beta$ whose relation is given by the Dynkin diagram of $\mathbf{G}_{2}$ as $(\alpha, \alpha)=3(\beta, \beta)$. So we can normalize by setting $\left(\varepsilon_{1}, \varepsilon_{1}\right)=1$ and then compute gradually the structural constants from the relation $N_{\alpha, \beta}^{2}=\frac{q(p+1)}{2}(\alpha, \alpha)$ where $p, q$ are the largest nonnegative integers such that $\beta+\kappa \alpha$ is a root $(-p \leq \kappa \leq q)$. From Proposition 3 we obtain

Proposition 10. For the space $G_{2} / U(2)$ the Einstein equation reduces to the following system of three equations and three unknowns $g_{1}, g_{2}, g_{3}$ :

$$
\begin{aligned}
& \operatorname{Ric}\left(E_{\varepsilon_{1}}, E_{-\varepsilon_{1}}\right) \\
& =1+\frac{3}{2}+\frac{1}{4}\left(\frac{3}{g_{2} g_{3}}\left(g_{1}^{2}-\left(g_{2}-g_{3}\right)^{2}\right)+\frac{2}{g_{1} g_{2}}\left(g_{1}^{2}-\left(g_{1}-g_{2}\right)^{2}\right)\right)=g_{1}, \\
& \operatorname{Ric}\left(E_{\varepsilon_{1}+\varepsilon_{2}}, E_{-\left(\varepsilon_{1}+\varepsilon_{2}\right)}\right)=1+\frac{1}{4}\left(\frac{6}{g_{1} g_{3}}\left(g_{2}^{2}-\left(g_{1}-g_{3}\right)^{2}\right)+4 \frac{g_{2}^{2}}{g_{1}^{2}}\right)=g_{2}, \\
& \operatorname{Ric}\left(E_{2 \varepsilon_{1}+\varepsilon_{2}}, E_{-\left(2 \varepsilon_{1}+\varepsilon_{2}\right)}\right)=3+\frac{3}{2}+\frac{1}{4}\left(\frac{3}{g_{1} g_{2}}\left(g_{3}^{2}-\left(g_{1}-g_{2}\right)^{2}\right)\right)=g_{3} .
\end{aligned}
$$

Theorem 11. The space $G_{2} / U(2)$ admits (up to scale) precisely three Einstein metrics. One is Kähler, given by $g_{1}=1, g_{2}=2, g_{3}=3$. The two non-Kähler 
Einstein metrics are given by $g_{1}=1, g_{2}, g_{3}$, where $g_{2}$ and $g_{3}$ are the two positive (real) solutions of the system

$$
\left\{\begin{array}{l}
3 g_{3}^{2}-9 g_{2}^{2}-6 g_{2}^{2} g_{3}+20 g_{2} g_{3}-16 g_{3}+9=0 \\
9 g_{3}^{2}-9 g_{2}^{2}-4 g_{2}^{2} g_{3}+24 g_{2}-16 g_{3}+3=0
\end{array}\right.
$$

Notice that in the examples (C) and (D) the normal metric is not Einstein.

The Kähler-Einstein metrics. There are two ways to decide which of the metrics presented above are Kähler-Einstein. The first is the independent calculation from the formula $g_{\alpha}=k(\alpha, \delta), \alpha \in R_{M}^{+}$, where $\delta$ is half the sum of the positive roots in $R_{M}$. We refer to [B-F-R, pp. 626-627] for a proof of this formula.

For the second, we can use the following necessary and sufficient condition for the metric $g$ in Proposition 1 to be Kähler with respect to a choice of an invariant complex structure. According to [Alek] the metric $g$ is Kähler if and only if the numbers $g_{\alpha}$ satisfy the relations

$$
g_{\alpha}+g_{\beta}=g_{\alpha+\beta}, \quad \alpha, \beta, \alpha+\beta \in R_{\mathfrak{t}}^{+},
$$

(we also refer to [A] for a detailed proof of this relation).

It is easy to check which of the solutions presented above satisfy these relations, so we can identify the Kähler-Einstein metrics. It is also possible to simplify the systems of Propositions $4,6,8$, and 10 by using the relations $g_{\alpha}+g_{\beta}=g_{\alpha+\beta}$, in which case the solutions of the new systems are the KählerEinstein metrics.

\section{ISOMETRIC AND NONISOMETRIC EINSTEIN METRICS}

Here we examine which of the Einstein metrics presented in $\S 5$ are isometric.

According to the variational approach to Einstein metrics, the critical points of the total scalar curvature functional $T(g)=\int_{M} S(g) d \operatorname{vol}(g)$ on the space of Riemannian metrics of Volume 1, are precisely the Einstein metrics. In this case the Einstein constant $c$ in the equation $\operatorname{Ric}(g)=c g$ is equal to $S(g) / n$ $(n=\operatorname{dim} M)$ [Mu, p. 522]. Thus if two Einstein metrics with volume 1 have different Einstein constants, then they can not be isometric. So in the following, when we say that we compute the Einstein constants, we mean that we take the explicit solutions of the Einstein equations, normalize the volume, and then calculate the Einstein constants from (any of) the equations.

We now turn to our examples. If the integers $n_{1}, n_{2}, n_{3}$ are distinct, then the metrics of Theorem 5 are not isometric since their Einstein constants are different. The volume is given by $g_{12}^{2 n_{1} n_{2}} g_{13}^{2 n_{1} n_{3}} g_{23}^{2 n_{2} n_{3}}$. Notice though, that if $n_{1}=n_{2}$ (say) then we get Alekseevsky's Proposition 2.3 [Alek], where the metrics (a) and (c) are isometric.

In Theorem 7 we gave three classes of Einstein metrics: the normal metric, the $n ! / 2$ Kähler-Einstein metrics, and the $n$ new metrics. The volume is given by $\prod g_{i j}^{2}$, and we get the same Einstein constants only among the KählerEinstein metrics and among the new metrics. All the Kähler-Einstein metrics are isometric via automorphisms of $S U(n) / T, T$ a maximal torus, which correspond to elements of the Weyl group of $S U(n)$, and the same is true for the new metrics. 
In Theorem 9 the volume is given by $f^{2 m^{2}} g^{2 m^{2}} h_{1}^{m(m-1)} h_{2}^{m(m-1)}$, and we get the same Einstein constants for the pairs of metrics (a)-(b), (c)-(e), and (d)(f) as well as for the Kähler-Einstein metrics (g), (h), (i) and (j). All these constants are different among themselves. Now, there is an automorphism of $S O(2 n) / U(m) \times U(m)$ corresponding to an element of the Weyl group of $S O(2 n)$ which has the effect (on an invariant metric) of switching $h_{1}$ and $h_{2}$. There is an automorphism corresponding to an outer automorphism of $S O(2 n)$ which has the effect of switching $f$ and $g$. Hence, any two of our invariant Einstein metrics which have the same volume are isometric via such automorphisms. So for $S O(2 n) / U(m) \times U(m)$ we obtain four nonisometric invariant Einstein metrics, one of which is Kähler.

Finally, by approximating the Einstein constants numerically in Theorem 11, we see that they are all different.

\section{REFERENCES}

[A] A. Arvanitoyeorgos, Invariant Einstein metrics on homogeneous spaces, Ph.D. thesis, University of Rochester, 1991.

[Alek] D. V. Alekseevsky, Homogeneous Einstein metrics, Differential Geometry and its Applications (Proceedings of Brno Conference), Univ. of J. E. Purkyne-Czechoslovakia, 1987, pp. $1-21$.

[Alek-Pe] D. V. Alekseevsky and A. M. Perolomov, Invariant Kähler-Einstein metrics on compact homogeneous spaces, Funct. Anal. Appl. 20 (1986), 171-182.

[Be] A. L. Besse, Einstein manifolds, Springer-Verlag, Berlin, 1985.

[B-F-R] M. Bordemann, M. Forger, and H. Römer, Homogeneous Kähler manifolds: paving the way towards new supersymmetric sigma models, Comm. Math. Phys. 102 (1986), 605-647.

[Gue] M. A. Guest, Geometry of maps between generalized flag manifolds, J. Differential Geometry 25 (1987), 223-247.

[Ko-No] S. Kobayashi and K. Nomizu, Foundations of differential geometry. II, Wiley, New York, 1969.

[Ki] M. Kimura, Homogeneous Einstein metrics on certain Kähler $C$-spaces, Adv. Stud. Pure Math. 18-I (1990), 303-320.

[Mu] Y. Muto, On Einstein metrics, J. Differential Geometry 9 (1974), 521-530.

[Sa] A. Sagle and R. Walde, Introduction to Lie groups and Lie algebras, Academic Press, New York and London, 1973.

[Var] V. S. Varadarajan, Lie groups, Lie algebras and their representations, Prentice-Hall, Englewood Cliffs, N. J., 1974.

[Wal] N. R. Wallach, Compact homogeneous Riemannian manifolds with strictly positive curvature, Ann. of Math. 96 (1972), 227-295.

[W] Z-X Wan, Lie algebras, Pergamon Press, Oxford, 1975.

[Wa] H. C. Wang, Closed manifolds with homogeneous complex structures, Amer. J. Math. 76 (1954), 1-32.

[Wa-Zi] M. Wang and W. Ziller, On normal homogeneous Einstein metrics, Ann. Sci. Ecole Norm. Sup. 18 (1985), 563-633.

Department of Mathematics, University of Rochester, Rochester, New York 14627 Current address: Filis 181, 11252 Athens, Greece

E-mail address: isambo@grathun 1 